\title{
A simplified method to calculate telomere length from Southern blot images of terminal restriction fragment lengths
}

Lisa F Lincz ${ }^{\star 1,3,4,5}$, Fiona E Scorgie ${ }^{1,4,5}$, Madhu B Garg ${ }^{2,4,5}$, Jayne Gilbert ${ }^{2,4,5}$ \& Jennette A Sakoff2,4,5,6

\section{ABSTRACT}

Southern blotting of DNA terminal restriction fragment lengths is the gold standard for measuring mean telomere length. Analysis of the final image is a crucial step in this process, however, current techniques are cumbersome and prone to error. Here we present a simple and accurate method for analyzing telomere smears. Basic 2D gel imaging software was used to automatically subtract background, generate standard curves and calculate net intensity and MW at each position (i) along the telomere smear. Our method required no statistical software or major data manipulation and correctly classified $>80 \%$ of 18 samples as having short, medium or long telomeres compared with $33-72 \%$ using other methods.

\section{METHOD SUMMARY}

Here we demonstrate a new method of analysis to calculate mean telomere length from Southern blot images of DNA terminal restriction fragment lengths using basic imaging software. ImageQuant was used to automatically subtract background, generate standard curves and set MW across the width of the image. A series of 31-60 small (5 pixel) boxes were manually inserted along the length of each telomere smear to enable the software to calculate net intensity and $M W$ at each position (i). Then, the data were batch exported into Microsoft Excel for final calculation of mean telomere length.

\section{KEYWORDS}

cancer cell lines $\cdot$ mean telomere length $\cdot$ net intensity $\cdot$ peripheral blood $\cdot$ Southern blot - terminal restriction fragment length

${ }^{1}$ Haematology Department, Calvary Mater Newcastle, Australia; ${ }^{2}$ Medical Oncology Department, Calvary Mater Newcastle, Australia; ${ }^{3}$ School of Biomedical Sciences \& Pharmacy, University of Newcastle, Callaghan, Australia; ${ }^{4}$ Hunter Medical Research Institute, New Lambton, Australia; ${ }^{5}$ Hunter Cancer Research Alliance, NSW, Australia; ${ }^{6}$ School of Environmental \& Life Sciences (Chemistry), Faculty of Science, University of Newcastle, Callaghan, Australia; *Author for correspondence: Lisa. lincz@calvarymater.org.au

BioTechniques 68: 28-34 (January 2020) 10.2144/ btn-2019-0082
Human telomeres are protective nucleoprotein structures consisting of 5-15 kb of tandem TTAGGG repeats that cap the ends of chromosomes to help maintain genomic stability $[1,2]$. Telomeres progressively shorten by approximately $50 \mathrm{bp}$ with each cell division in normal cells $[3,4]$; however, they can also be negatively affected by environmental, genetic and lifestyle factors [5]. Once a critical telomere length is reached, cells are no longer able to divide and senescence ensues $[6,7]$. Accordingly, telomere length is often referred to as a mitotic clock and used as a biomarker of cellular aging and risk of related diseases [8].

Because telomere length varies between chromosomes, cells and tissues within any individual, obtaining an accurate measurement can be challenging. Although advanced molecular techniques have been developed, the original method of terminal restriction fragment length (TRFL) analysis remains the gold standard for quantitation of mean telomere length (MTL) $[3,9]$. The method uses restriction enzymes to digest gDNA and to leave behind intact telomere repeats, which are then resolved by agarose gel electrophoresis and visualized by hybridization to a labeled telomerespecific oligonucleotide probe. The intensity and size distribution of the resulting telomeric smear are used to calculate the MTL of the cell population. The first publication of this technique used traditional Southern blotting methodology. Based on the assumption of equal efficiency of DNA transfer at all areas of the gel, and with the number of telomere repeats per DNA fragment being proportional to the DNA length, calculated MTL by the Equation 1:

$$
\sum \mathrm{NI}_{\mathrm{i}} / \sum\left(\mathrm{NI}_{\mathrm{i}} / \mathrm{MW}_{\mathrm{i}}\right)
$$

where $\mathrm{NI}_{\mathrm{i}}$ is the net intensity at position (i) and $\mathrm{MW}_{\mathrm{i}}$ is the $\mathrm{MW}$ at position (i) [9]. Later publications using in-gel hybridization revised the calculation to Equation 2 [3]:

$$
\sum\left(\mathrm{NI}_{\mathrm{i}} \times \mathrm{MW}_{\mathrm{i}}\right) / \sum \mathrm{NI}_{\mathrm{i}}
$$

However, both equations remain in use today. In the last 10 years, detailed protocols describing the methodology and analysis procedures have been published and are still extensively referenced $[10,11]$. However, calculated MTL does not always reflect the apparent size distribution of telomeric smears depicted in hybridized images [9,11-13].

Although the laboratory procedures for generating and visualizing telomeric TRFL are generally straightforward and reproducible by a skilled molecular scientist, the manual image analyses described in current protocols are cumbersome, laborious, require knowledge, use of statistical software and fraught with potential for human error [10-12]. All strategies require conversion of distance (in pixels) to MW, using data that must be extracted from the image analysis and transferred to a separate software package to generate a standard curve. This must be repeated for each side of the image to account for any gel curvature, and then background removal is performed manually for each $\mathrm{NI}$ at every MW position before summing the required data for use in the MTL calculations. The goal of the present study was to simplify the TRFL image analysis so that a laboratory technician could 
perform the MTL calculations using basic imaging software and batch export of data to Microsoft Excel, so that all steps could be easily traceable and accounted for.

\section{METHODS}

\section{Samples}

Cell lines consisted of carcinomas derived from breast: BT20, MCF10A, T47D, MCF7, MDA-MB-231, MDA-MB-468,ZR-75-1, BT-474; colon: HT29; prostate: DU 145; pancreas: MIA; epidermis: A431; ovary: A2780; lung: $\mathrm{H} 460$; and neuroblastoma: $\mathrm{BE}(2)-\mathrm{C}$. All cell lines were obtained from the American Type Culture Collection (VA, USA) except for A431 and $A 2780$, which were purchased from the European Collection of Authenticated Cell Cultures (Public Health England, Salisbury, UK), and MDA-MB-468 and SK-BR-3, which were kind gifts from Michael Agrez and Judith Weidenhofer, respectively. All cells were maintained in Dulbecco's Modified Eagle Media (Sigma, MO, USA) supplemented with $10 \%$ fetal bovine serum at $37^{\circ} \mathrm{C}$ and $5 \% \mathrm{CO}_{2}$. Peripheral blood (PB) samples were collected from three healthy volunteers (PB1, 48-year-old male; PB2, 40-year-old female; PB3, 74-year-old male) after receiving informed consent. The study was approved by the Hunter New England Human Research Ethics Committee.

\section{Southern blotting}

DNA was isolated by the mini salt extraction method [14] and digested overnight at $37^{\circ} \mathrm{C}$ with $5 \mathrm{U}$ each of Hinfl, Rsal, Mspl, Hhal, Haelll and Alul [11] (Promega, WI, USA). Traditional Southern blotting was performed using reagents purchased from Roche (Roche Diagnostics, Mannheim, Germany) and a digoxigenin-labeled telomere probe (CCCTAA ${ }_{3}$; ECL Direct Nucleic Acid protocol, GE Healthcare UK Ltd, Little Chalfont, UK). Chemiluminescence images were captured on an Amersham Imager 600 RGB (GE Healthcare Bio-Sciences, Uppsala, Sweden), and they were analyzed using ImageQuant TL v8.2 software (GE Healthcare).

\section{Mean telomere length quantitation Our method}

The image was opened within the '1D gel analysis' utility of the ImageQuant TL software (Figure 1). The 'stepwise' setting enabled manual (as opposed to automatic) setup of the analysis. The number of lanes was manually set and drawn using the 'create lanes' function. Individual lanes were adjusted using the 'edit single lane' function as required. Background subtraction was performed automatically using the 'rubber band' setting. The band width was manually set to 5 pixels and the pen tool was used to identify the bands in the DNA marker lanes (lambda/HindIII ladder) by manually clicking on each. The 'current lane' view was used to identify and mark the beginning and end of the telomere smear in each sample lane. A series of adjacent 5-pixel boxes were then added between these boundaries along the length of the smear. When all of the lanes were marked in this way, the next analysis page was used to set the DNA MW by highlighting each DNA marker lane and selecting the appropriate DNA marker from the drop-down menu (we used Lam-Hin3). Individual marker lanes were then connected using the hand tool to identify each marker lane, resulting in horizontal lines being drawn between individual matching DNA MW markers and enabling the software to adjust for any distortions across the gel. The curve type was set to "cubic spline" and automatically computed by the software. The measurement window was then used to export all lane data into a text file, which was imported into Microsoft Excel, producing columns of individual pixel boxes (band number), corresponding net intensity (volume $=\mathrm{NI}$ ) and relative distance traveled (base pairs $=\mathrm{MW}$ ). Basic Microsoft Excel functions were used to generate columns of $\mathrm{NI} \times \mathrm{MW}$ and $\mathrm{NI} / \mathrm{MW}$ for each corresponding 5-pixel box (representing position [i]), and these were respectively summed to generate $\Sigma\left(\mathrm{NI}_{\mathrm{i}} \mathrm{xMW} \mathrm{W}_{\mathrm{i}}\right)$ and $\Sigma\left(\mathrm{NI}_{\mathrm{i}} /\right.$ $\mathrm{MW}_{\mathrm{i}}$ ) and used in the appropriate MTL equations:

$$
\begin{aligned}
& \text { MTL1 }=\sum \mathrm{NI}_{\mathrm{i}} / \sum\left(\mathrm{NI}_{\mathrm{i}} / \mathrm{MW}_{\mathrm{i}}\right) \text { and } \\
& \text { MTL2 }=\sum\left(\mathrm{NI}_{\mathrm{i}} \times \mathrm{MW}_{\mathrm{i}}\right) / \sum \mathrm{NI}_{\mathrm{i}}
\end{aligned}
$$

\section{Jenkins et al.}

This is a recently published modification of Mender \& Shay [11] (see below) [12], in which a grid of 150 boxes is positioned over the entire lane, but only the ones over the telomere smear were used for calculations. MTL1 is the suggested equation.

\section{Mender \& Shay}

Using the 'Toolbox' utility of ImageQuant $T L$, a grid of 150 boxes was positioned over the entire sample lane and used to calculate telomere length, after subtracting the intensity of an empty sample lane (background) at each point. Prism software (v8.0.2 GraphPad Software, CA, USA) was used to graph MW markers versus distance in pixels from sample well and fit a nonlinear regression curve with one phase exponential decay. This curve was divided into 150 data points to correspond with the 150 boxes covering each sample lane, and MW was calculated for each to enable calculation of NI/MW for each point (i) over the length of each lane. This process was repeated using separate MW marker lanes for each half of the gel to account for any curvature. MTL1 is the suggested equation [11].

\section{Kimura et al.}

In this first published detailed protocol [10], each lane was exported individually as a pixel versus intensity profile into Microsoft Excel, providing an intensity reading for every single pixel distance from the sample well to the bottom of the lane, generating over 1500 data points per sample. This was achieved in the 1D gel analysis utility of ImageQuant TL. Background removal from each point using intensity measured from an empty sample lane was performed manually in Microsoft Excel. The pixel profile was used to determine the distance of each MW marker from the well, and a scatter graph of MW vs pixels was generated in Microsoft Excel. An exponential curve (power function) was fitted, and the corresponding equation was used to convert pixels to $\mathrm{MW}$ for each point of every sample. In the original method, the data were collected from 20,000 bp with a lower limit of 1200 or $3000 \mathrm{bp}$, depending on the age of the blood donors. In the current study, we modified this to the highest MW marker (23,130 bp) and extended the lower limit to the lowest marker (564 bp) considering the excessively short telomere lengths of the cell lines. To account for any curvature, this process was repeated using separate MW marker lanes for each half of the gel. MTL1 is the suggested equation. 


\section{Statistical analysis}

Telomere lengths were classified manually by visual assessment of the location of the telomere profile on the imaged blot: above the 4361 bp marker were considered 'long,' between 4361 and 2322 bp markers were considered 'medium' and the remainder were considered 'short.' Calculated MTL was expressed in bp, summarized as median [minimum (min)-maximum (max)] and analyzed as a continuous variable using nonparametric tests. The Wilcoxon matched pairs test was used to investigate individual differences in absolute MTL values, and Kendall coefficient of concordance (W) and Spearman rank order correlations were used to measure the strength and association of individual calculations based on their rankings. Variations between MTL results were calculated as \% coefficient of variance and the Kruskal-Wallis one-way analysis of variance used to compare effects of different amounts of starting DNA. All calculations were performed with Statistica software v10.0 (StatSoft, OK, USA) using two-tailed tests, and $p<0.05$ was considered statistically significant. Graphs were constructed using Prism software, version 8.0.2 (GraphPad Software, CA, USA).

\section{RESULTS \& DISCUSSION}

A total of 15 human cell lines and three peripheral blood samples were run on two separate blots (Supplementary Figure 1). Table 1 compares the results of our method to previously published analysis techniques [10-12] and MTL calculations using either equation MTL1 $=\Sigma \mathrm{NI}_{i} / \Sigma\left(\mathrm{Nl}_{\mathrm{i}} /\right.$ $\left.\mathrm{MW}_{\mathrm{i}}\right)$ or MTL2 $=\left(\mathrm{Nl}_{\mathrm{i}} \mathrm{XMW}_{\mathrm{i}}\right) / \Sigma \mathrm{NI}_{\mathrm{i}}$. Our method of using 5-pixel boxes along the telomere smear produced a similar number of data points as the method of Jenkins et al. [12], which was significantly less than the methods used by Kimura et al. [10] and Mender \& Shay [11], both of which measure $\mathrm{NI}$ along the complete lane, from the sample well to the bottom of the gel. ImageQuant software will not allow the 5-pixel boxes to be adjoined, resulting in intervals of 9-20 pixels between the boxes. We performed a separate analysis in which these gaps were omitted by painstakingly deleting one boundary from each box to effectively form a column of larger boxes (resulting in significantly fewer data points) along each smear and found no significant difference between the MTL results. However, this was only true when the analysis was limited to the telomere smear, because inclusion of any other areas outside of this (as used by both Kimura et al. [10] and Mender \& Shay [11]) significantly affected the final results (data not shown).

There was significant variation between methods when either respective equation was used to calculate MTL $(p<0.0001)$. In this regard, MTL1 produced significantly shorter measurements compared with MTL2 $(\mathrm{p}<0.0001)$. Mathematically, MTL1 $=\Sigma \mathrm{NI}_{\mathrm{i}} /$ $\Sigma\left(\mathrm{NI}_{\mathrm{i}} / \mathrm{MW}_{\mathrm{i}}\right)$ is a harmonic mean, whereas MTL2 $=\left(\mathrm{Nl}_{\mathrm{i}} \mathrm{XMW}_{\mathrm{i}}\right) / \Sigma \mathrm{NI}_{\mathrm{i}}$ is an arithmetic mean. Both have the added level of MW, which, in the case of MTL2, results in higher data points being given greater weights than lower data points, resulting in an underrepresentation of low-MW fragments that also have reduced intensity compared with larger fragments. Importantly, the dynamic range (ratio of the highest to the lowest calculated value) of MTL was also consistently lower (1.73-2.77) using MTL1 compared with 2.69-3.74 using MTL2.

Figure 2 illustrates the individual MTL results calculated for each sample. There was significantly more variation in individual sample telomere lengths calculated using MTL2, with an average \% coefficient of variance of $67.49 \%$, compared with $18.51 \%$ when MTL1 was used (data not shown). Most of the disparity in the MTL2 results originated from the analysis using the Mender \& Shay method, as the variation was reduced to $17.39 \%$ when these results were omitted. Because the same raw $\mathrm{NI}$ and $\mathrm{MW}$ values were used in both of the MTL1 and MTL2 calculations, the mathematical differences (as described above) are the main cause for discrepancy in this instance. However, the greatly exaggerated MTL2 values obtained using the Mender \& Shay method were not replicated when the modified Jenkins et al. technique was used, indicating that the inclusion of areas outside of the telomere smear was most likely falsely contributing to the calculated MTL. Curiously, this was not as pronounced in the results obtained using the Kimura et al. method, which similarly measures $\mathrm{NI}$ along much of the sample lane. The main difference between these two methods is the size of the $\mathrm{Nl}$ area being measured at each position (i). Examination of the raw $\mathrm{NI}_{i}$ values confirmed inadequate background removal from the larger areas of measurement used by Mender \& Shay, as compared with the individual pixel points employed by Kimura et al.

Table 1. Results of telomere lengths calculated using four different analysis methods and applying two different equations.

\begin{tabular}{|c|c|c|c|c|}
\hline & $\begin{array}{l}\text { Data points }(\mathbf{n}) \\
\text { Median (min-max) }\end{array}$ & $\begin{array}{l}\text { MTL1 }(b p)=\Sigma N_{i} / \Sigma\left(N_{i} / M W_{i}\right) \\
\text { Median }(\min -\max )\end{array}$ & $\begin{array}{l}\operatorname{MTL} 2(\mathbf{b p})=\Sigma\left(\mathrm{NI}_{\mathrm{i}} \times \mathrm{MW}_{\mathrm{i}}\right) / \Sigma \mathrm{NI}_{\mathrm{i}} \\
\text { Median }(\min -\max )\end{array}$ & $\begin{array}{l}\text { MTL1 vs MTL2 } \\
\text { p-value }\end{array}$ \\
\hline $\begin{array}{l}\text { (a) Our method } \\
\text { MTL dynamic range }\end{array}$ & $42(31-60)$ & $\begin{array}{l}2802(1763-4891) \\
2.77\end{array}$ & $\begin{array}{l}3121(2145-7370) \\
3.44\end{array}$ & $<0.0001$ \\
\hline $\begin{array}{l}\text { (b) Jenkins et al. [12] } \\
\text { MTL dynamic range }\end{array}$ & $47(26-58)$ & $\begin{array}{l}2565(2228-5808) \\
2.61\end{array}$ & $\begin{array}{l}3272(2755-7656) \\
2.78 \\
\end{array}$ & $<0.0001$ \\
\hline $\begin{array}{l}\text { (d) Kimura et al. [10] } \\
\text { MTL dynamic range }\end{array}$ & $973^{\star \star}(761-1005)$ & $\begin{array}{l}2071 *(1763-4199) \\
2.38\end{array}$ & $\begin{array}{l}4083(2458-6619) \\
2.69\end{array}$ & $<0.0001$ \\
\hline
\end{tabular}

Methods significantly different compared with (a) are indicated by ${ }^{*} p<0.01$ and ${ }^{* *} \mathrm{p}<0.001$ (Wilcoxon matched pairs test).

bp: Base pair; max: Maximum; min: Minimum; MTL: Mean telomere length; NI: Net intensity. 


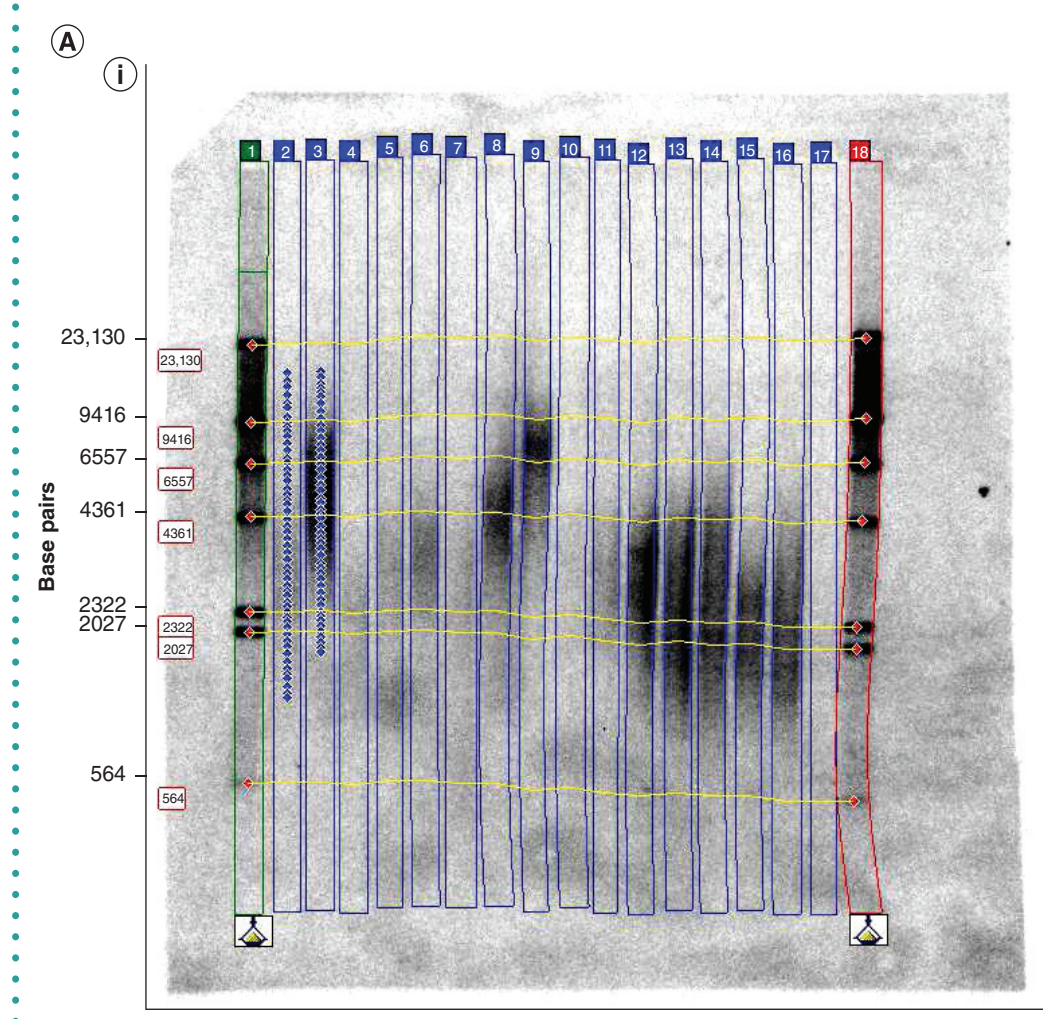

(ii)
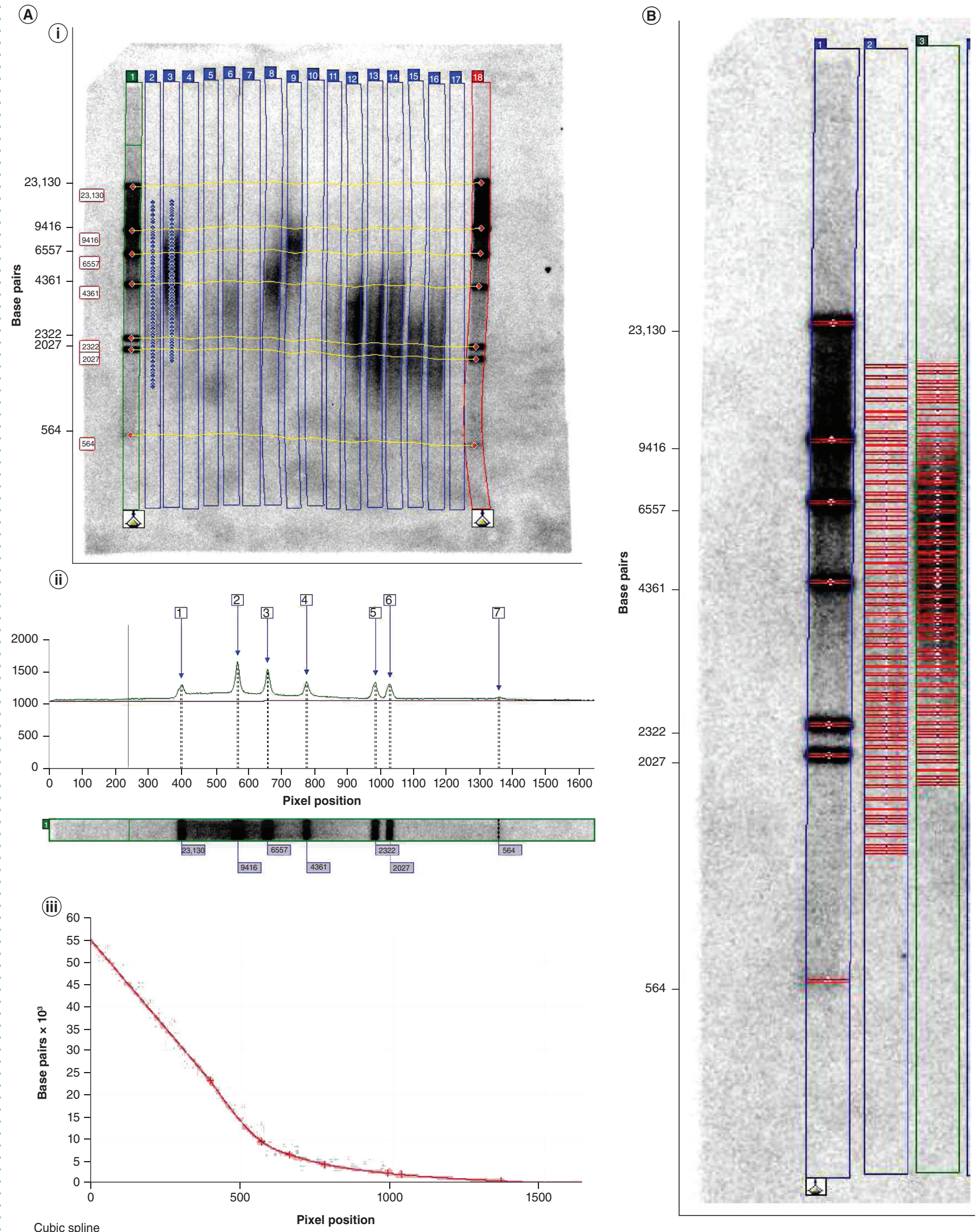

Formula: $n / a$

$R^{2}$ value: $n / a$ 


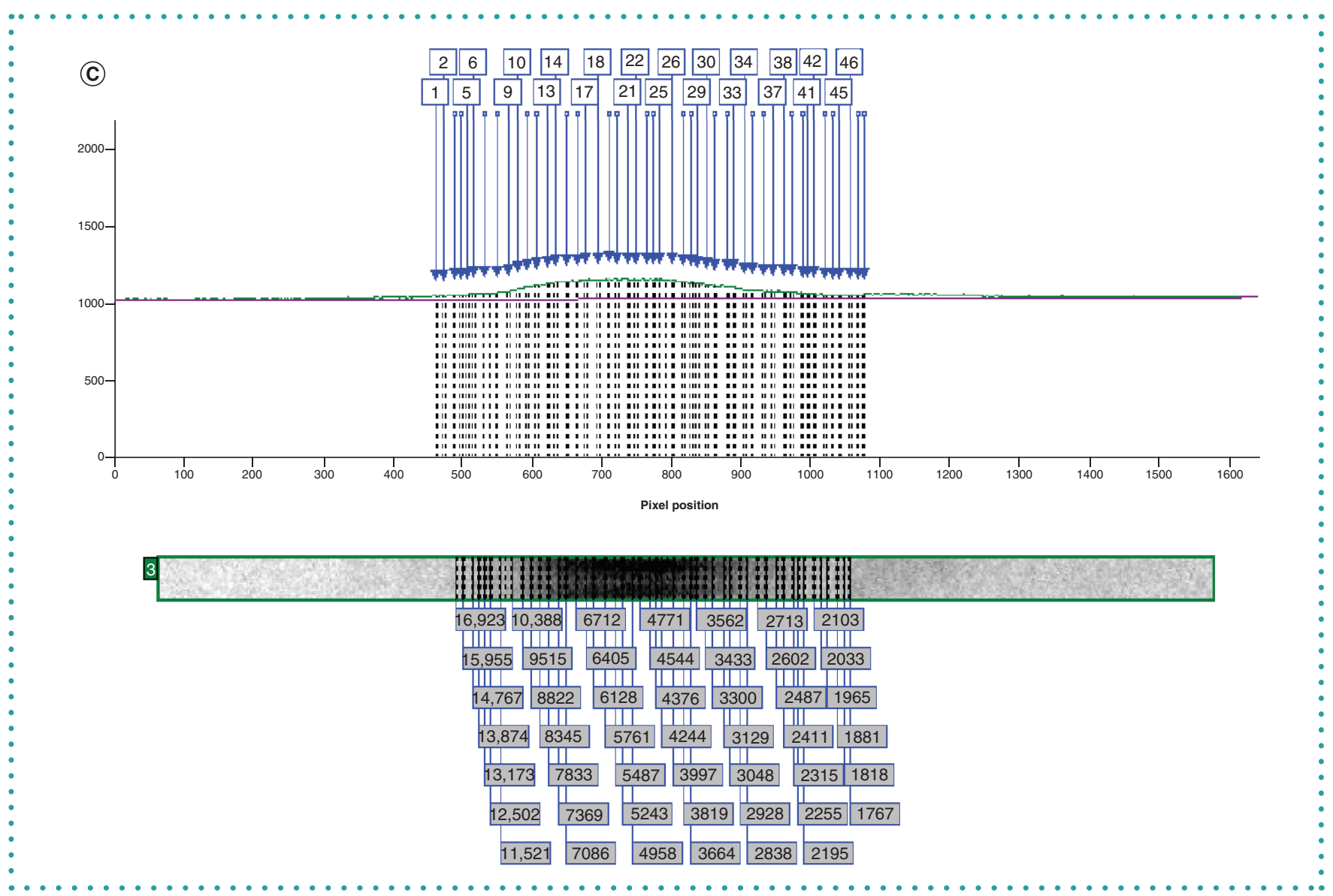

Figure 1. Analysis of terminal restriction fragment lengths in Southern blot images (also see facing page). (A) ImageQuant interface illustrating (i) lane detection and MW marker assignment in lanes 1 and 18 with connecting horizontal lines generated across the image in yellow, (ii) upper panel shows intensity profile of molecular weight markers in lane 1 with corresponding lane image in lower panel and (iii) corresponding software-generated cubic spline standard curve. (B) Magnified view of lanes 1, 2 and 3 from blot image in A(i) illustrating placement of 5-pixel boxes (red horizontal lines) along the telomere smears in lanes 2 and 3. (C) Upper panel shows intensity profile of corresponding lane 3 (in lower panel) with number and position of each pixel box along the telomere smear.

It is important to note that our method was the most accurate at reflecting the Southern blot visual results by correctly classifying samples as having short, medium or long telomeres using either MTL1 (16/18, $89 \%)$ or MTL2 (15/18, $83 \%)$ compared with the other methods, which ranged from $13 / 18$ to $6 / 18$ (72 to $33 \%)$, respectively. The accuracy of our method could be further improved to $100 \%$ by employing MTL1 for samples with telomere smears falling below 4361 bp and MTL2 for those above. MTL1 is technically the most correct formula to use for DNA movement through a gel, which is not linear but rather logarithmic. However, the exclusive use of MTL1 likely results in an underestimation of MTL in many human studies in which telomere lengths are likely to be $>4361 \mathrm{bp}$. Both equations are inherently flawed in that they assume that intensity is proportional to copy number, when in reality multiple copies of the $\mathrm{CCCTAA}_{3}$ probe can hybridize to the complementary telomeric TTAGGG repeat regions. Therefore, neither equation can provide an absolutely correct MTL measurement, but simply a much more accurate approximation than can be estimated by eye. Ideally, all calculated MTL values in this study should be compared with the "known" telomere length of the samples. However, there is currently no perfect method to provide an exact MTL, and, therefore, this study is limited in its ability to precisely measure the accuracy of the results. Although a more objective method of sample categorization would be preferred, we firmly believe that the final calculated MTL value should be consistent with what is illustrated on the blot.

Not surprisingly, the four different analysis methods were only moderately concordant in terms of ranking the samples from shortest to longest using either MTL1 $(\mathrm{W}=0.49)$ or MTL2 ( $\mathrm{W}=0.60$; data not shown). Individual correlation analysis revealed that the MTL results using our analysis method, and either MTL1 or MTL2, were most similar to those obtained using the combination of Jenkins et al.'s method with MTL1 $\left(r_{s}=0.769, p<0.001\right.$ and $r_{s}=0.810$, $\mathrm{p}<0.0001$, respectively). In contrast, the method of Mender \& Shay combined with MTL2 provided the most discordant results, producing non-significant and/or negative correlations with all of the other methods (Supplementary Table 1).

The robustness of our analysis was tested by varying the amounts of starting DNA added to the digest reaction. There was no significant difference in the results $(p=0.973)$ with variations of less than $1.5 \%$ across the calculated mean telomere lengths. This was comparable to results 


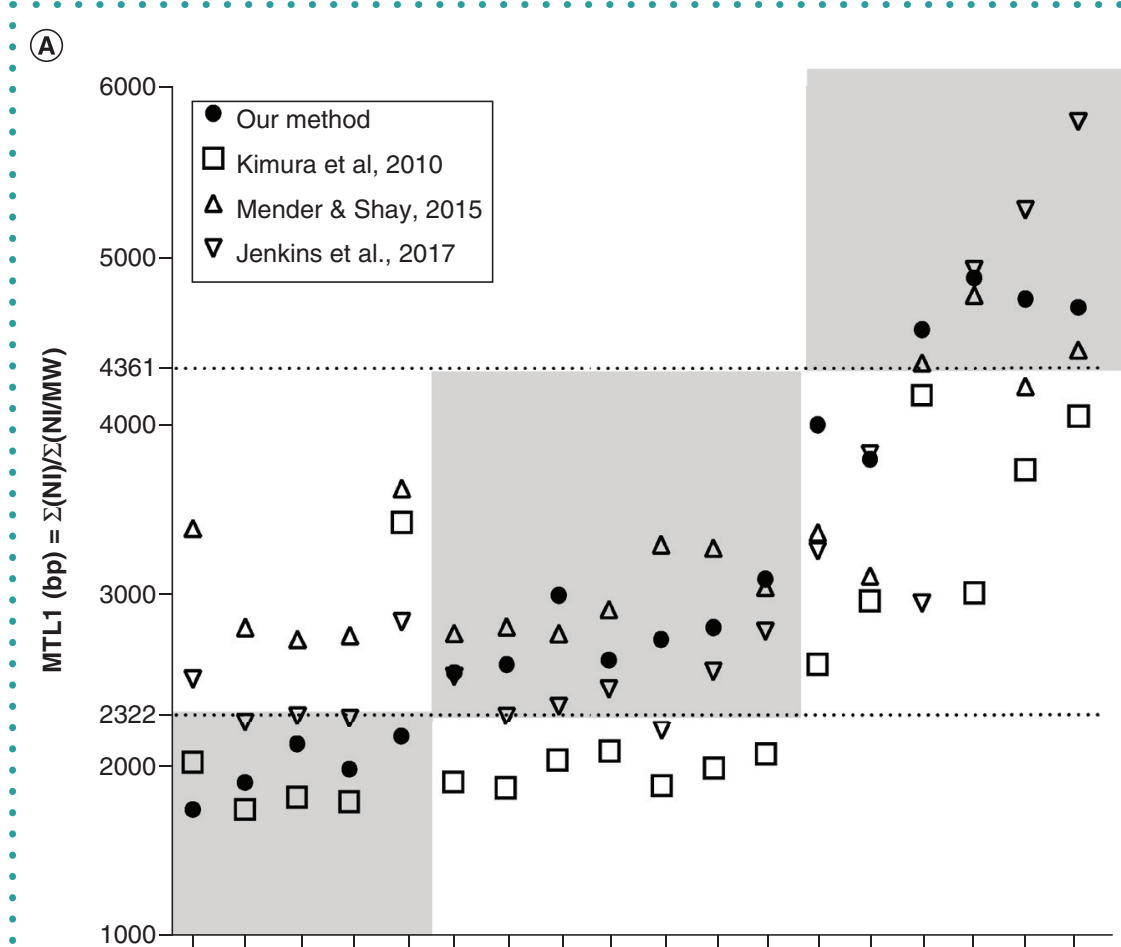

(B)

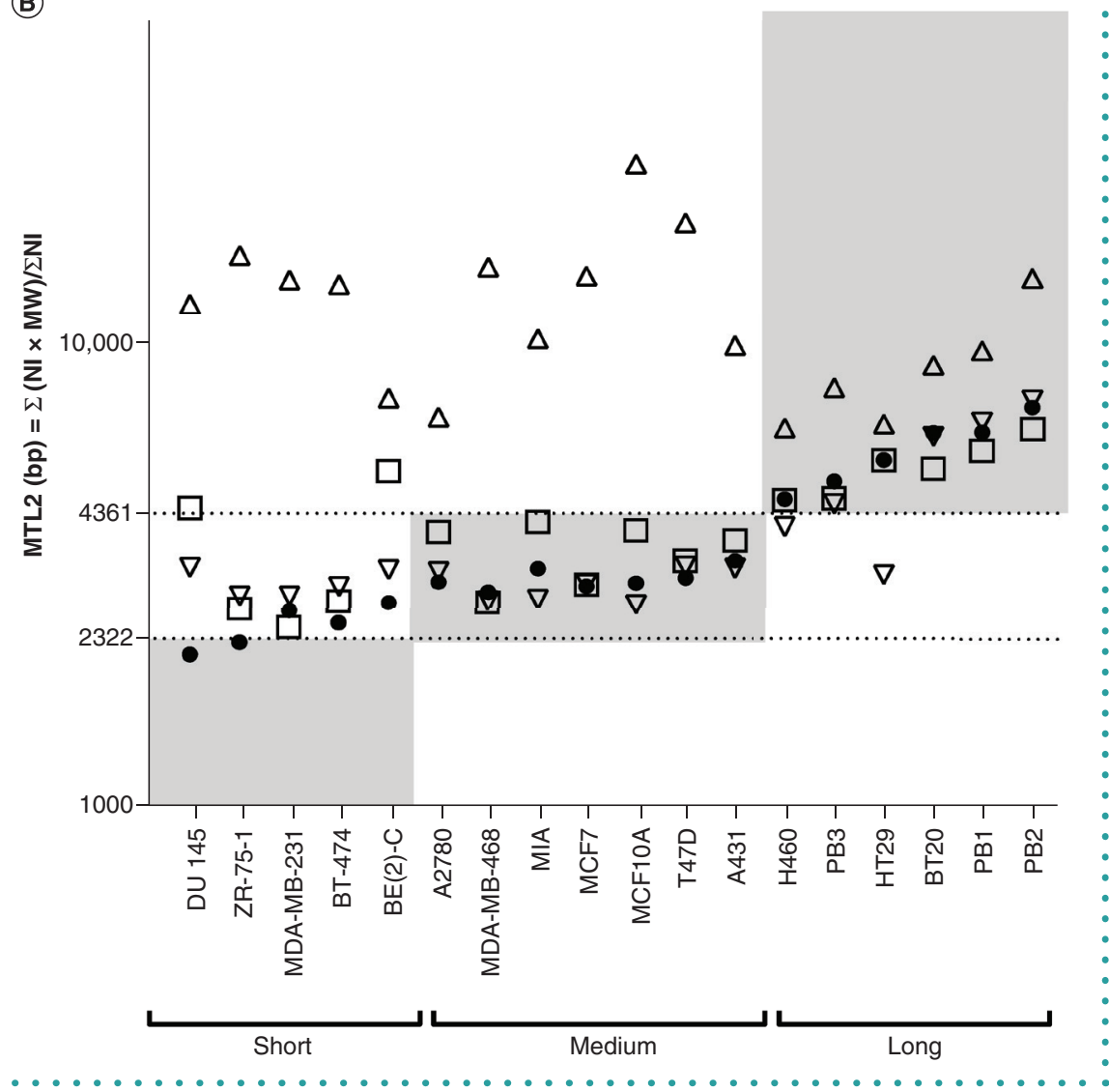

Figure 2. Comparison of individual mean telomere length results for samples with short, medium and long telomeres (indicated by the shaded regions) calculated using equation MTL1 (A) or MTL2 (B).

- Our method; $\boldsymbol{\nabla}$ Jenkins et al., 2017; $\Delta$ Mender \& Shay 2015; $\square$ Kimura et al. 2010. bp: Base pair; MTL: Mean telomere length; NI: Net intensity. using the other calculations (Supplementary Table 2).

In conclusion, we have developed a simplified method of TRFL image analysis that exploits the capabilities of any basic 2D gel analysis software to minimize the two main sources of error encountered in other manual analysis techniques: fitting a standard curve and removing interfering background. Our use of automated curve fitting for MW markers strategically placed on the gel allows the software to accurately calculate MW across the membrane. This technique accounts for any curvature that may occur during electrophoresis, and hence is more robust for use on less-thanperfect gels. Similarly, the use of automated background removal that is specific to each lane of the blot eliminates the generation of negative values encountered when a single blank lane is used as the background to be subtracted from all others. The number of data points required for each MTL calculation has been minimized to alleviate the unwieldiness of large data files for digital manipulation, and data transfer has been simplified by exporting all lanes as a single batch file rather than individually. In doing so, we have minimized the risk of human error by eliminating the need for copying and pasting of data into multiple programs. It is the culmination of all of these improvements (i.e., superior standard curve calculation, interpolation of marker positions across the blot, precise conversion of pixels to MW and increased accuracy of background subtraction), combined with the ease of data export and minimal statistical manipulation, that makes our method an attractive option for researchers performing manual calculation of mean telomere length by TRFL.

There are many procedures currently used for measuring telomere length: quantitative PCR, various adaptations of quantitative FISH, 'Single Telomere Length Analysis' and the recently developed 'Telomere Shortest Length Assay' [15]. Quantitative PCR and TRFL are arguably the most popular, as they require less specialization and are able to be performed using basic laboratory techniques and equipment. Although both are useful for analyzing large populations of cells, 
quantitative PCR delivers only a relative ratio of telomere to reference gene signal, whereas TRFL provides absolute quantification of MTL, allowing for comparisons between studies and laboratories. However, even this method is prone to interference from methodological variations, such as DNA extraction methods, selection of type and number of restriction enzymes, efficiency of DNA transfer and probe hybridization and acquisition of suitable images. These are accepted limitations that can be minimized by optimizing protocols and introducing appropriate measures of quality control $[11,15]$. Nevertheless, analysis of the final image remains a crucial step in the process of calculating MTL, and we have shown here that significant variation can be introduced depending on the methodology employed.

Although specific MTL calculation programs, such as TeloTool [16], TeloRun [17], and Telometric [18], have been developed by individual researchers and are freely available, these programs have not been largely embraced by the larger scientific community. In our experience, the interfaces were not user-friendly, and the software contained many glitches. Coupled with an inability to save previous work, completing any single analysis in one sitting was practically impossible. Therefore, many researchers rely on manual techniques, as evidenced by the high citation rates for the protocols investigated in this study [10-12].

\section{FUTURE PERSPECTIVE}

It is clear from the length of each telomeric smear that there is much heterogeneity within cell populations, and many have suggested that it is the shortest telomeres that are most relevant to human disease, as these are the ultimate triggers of cellular senescence [19]. Although TRFLs can be used to visualize short telomeres, it remains difficult to determine their relative proportion within a sample. The Telomere Shortest Length Assay is a promising new method that enables absolute quantification of mean telomere length as well as the percentage of the smallest telomeres [13]. However, as with many of the other techniques listed previously, the adaptability of this specialized procedure to general research labora- tories may prove challenging, leaving traditional methods such as TRFL with a constant presence in future scientific literature.

\section{SUPPLEMENTARY DATA}

To view the supplementary data that accompany this paper please visit the journal website at: www.future-science. com/doi/suppl/10.2144/btn-2019-0082

\section{AUTHOR CONTRIBUTIONS}

LF Lincz and JA Sakoff conceived the study and designed the experiments. MB Garg and $\mathrm{J}$ Gilbert were responsible for growing cell lines and collecting blood samples. FE Scorgie isolated the DNA and performed all Southern blotting experiments. LF Lincz analyzed the data and drafted the manuscript. All authors contributed to manuscript revision and approved the final version.

\section{ACKNOWLEDGMENTS}

The authors wish to thank the Hematology and Medical Oncology Units of the Calvary Mater Newcastle for their ongoing support of our research.

\section{FINANCIAL \& COMPETING INTEREST DISCLOSURE}

Funding for this study was provided through the Mark Hughes foundation, Newcastle, Australia. The authors have no other relevant affiliations or financial involvement with any organization or entity with a financial interest in or financial conflict with the subject matter or materials discussed in the manuscript apart from those disclosed.

No writing assistance was utilized in the production of this manuscript.

\section{ETHICAL CONDUCT OF RESEARCH}

The authors state that they have obtained appropriate institutional review board approval and have followed the principles outlined in the Declaration of Helsinki for all human investigations. In addition, informed consent has been obtained from the participants involved.

\section{OPEN ACCESS}

This work is licensed under the AttributionNonCommercial-NoDerivatives 4.0 Unported License. To view a copy of this license, visit http://creativecommons.org/licenses/ by-nc-nd/4.0/

\section{REFERENCES}

Papers of special note have been highlighted as:

.. of considerable interest

1. Moyzis RK, Buckingham JM, Cram LS et al. A highly conserved repetitive DNA sequence, (TTAGGG)n, presconserved repetitive DNA sequence, (TTAGGG)n, presNatl Acad. Sci. USA 85(18), 6622-6626 (1988).

2. Samassekou O, Gadji M, Drouin R, Yan J. Sizing the ends: normal length of human telomeres. Ann. Anat. 192(5), 284-291 (2010).

3. Vaziri $H$, Schachter F, Uchida I et al. Loss of telomeric DNA during aging of normal and trisomy 21 human lymphocytes. Am. J. Hum. Genet. 52(4), 661-667 (1993).

4. Hastie ND, Dempster M, Dunlop MG, Thompson AM, Green DK, Allshire RC. Telomere reduction in human colorectal carcinoma and with ageing. Nature 346(6287), 866-868 (1990).

5. Shammas MA. Telomeres, lifestyle, cancer, and aging. Curr. Opin. Clin. Nutr. Metab. Care 14(1), 28-34 (2011).

6. Allsopp RC, Vaziri H, Patterson C et al. Telomere length predicts replicative capacity of human fibroblasts. Proc. Natl Acad. Sci. USA 89(21), 10114-10118 (1992).

7. Hayflick L, Moorhead PS. The serial cultivation of human diploid cell strains. Exp. Cell Res. 25, 585-621 (1961).

8. Smith L, Luchini C, Demurtas J et al. Telomere length and health outcomes: an umbrella review of sysand health outcomes. an umbrella review of sysstudies. Ageing Res. Rev. 51, 1-10 (2019).

9. Harley $C B$, Futcher $A B$, Greider CW. Telomeres shorten during ageing of human fibroblasts. Nature 345(6274), 458-460 (1990).

10. Kimura M, Stone RC, Hunt SC et al. Measurement of telomere length by the Southern blot analysis of terminal restriction fragment lengths. Nat. Protoc. 5(9), 1596-1607 (2010).

.. This represents the first publication of a comprehensive TRFL analysis protocol that is arguably still the sive TRFL analysis proto
most widely cited today.

11. Mender I, Shay JW. Telomere Restriction Fragment (TRF) Analysis. Bio. Protoc. 5(22), e1658 (2015).

. This is the second published protocol for TRFL analysis. It is not as popular as that of Kimura et al., 2010, but represents a different analysis approach that we felt was important to examine in our study.

12. Jenkins FJ, Kerr CM, Fouquerel E, Bovbjerg DH Opresko PL. Modified terminal restriction fragment analysis for quantifying telomere length using in-gel hybridization. J. Vis. Exp. 125, e56001 (2017).

.. This is the third and latest publication of a TRFL analysis protocol. It is a modified version of Mender \& Shay (2015), but its recent publication indicates that his (2015), but its recent publication indicates that this area of research is still very topical; therefore, study.

13. Lai TP, Zhang N, Noh J et al. A method for measuring the distribution of the shortest telomeres in cells and tissues. Nat. Commun. 8(1), 1356 (2017).

14. Miller SA, Dykes DD, Polesky HF. A simple salting out procedure for extracting DNA from human nucleated cells. Nucleic Acids Res. 16(3), 1215 (1988).

15. Lai TP, Wright WE, Shay JW. Comparison of telomere length measurement methods. Philos. Trans. R. Soc Lond. B. Biol. Sci. 373(1741), (2018).

16. Gohring J, Fulcher N, Jacak J, Riha K. TeloTool: a new tool for telomere length measurement from terminal restriction fragment analysis with improved probe intensity correction. Nucleic Acids Res. 42(3), e21 (2014).

17. Baur JA, Wright WE, Shay JW. Analysis of mammalian telomere position effect. Methods Mol. Biol. 287. 121-136 (2004)

18. Grant JD, Broccoli D, Muquit M, Manion FJ, Tisdall J, Ochs MF. Telometric: a tool providing simplified, reproducible measurements of telomeric DNA from constant field agarose gels. Biotechniques 31(6), 1314-1316, 1318 (2001).

19. Hemann MT, Strong MA, Hao LY, Greider CW. The shortest telomere not average telomere length is critical for cell viability and chromosome stability. Cell 107(1), 67-77 (2001). 